\title{
Vortex-mediated relaxation of magnon BEC into light Higgs quasiparticles
}

\author{
S. Autti $\odot,{ }^{1,2,{ }^{*}}$ P. J. Heikkinen $\odot,{ }^{1,3}$ S. M. Laine, ${ }^{4}$ J. T. Mäkinen $\odot,{ }^{1,5,6}$ E. V. Thuneberg $\odot,{ }^{4,7}$ \\ V. V. Zavjalov, ${ }^{1,2}$ and V. B. Eltsov (10 ${ }^{1}$ \\ ${ }^{1}$ Department of Applied Physics, Aalto University, P.O. Box 15100, FI-00076 AALTO, Finland \\ ${ }^{2}$ Department of Physics, Lancaster University, Lancaster LA1 4YB, United Kingdom \\ ${ }^{3}$ Department of Physics, Royal Holloway, University of London, Egham, Surrey TW20 OEX, United Kingdom \\ ${ }^{4}$ Nano and Molecular Systems Research Unit, University of Oulu, P.O. Box 3000, FI-90014 Oulu, Finland \\ ${ }^{5}$ Department of Physics, Yale University, New Haven, Connecticut 06520, USA \\ ${ }^{6}$ Yale Quantum Institute, Yale University, New Haven, Connecticut 06520, USA \\ ${ }^{7}$ Department of Applied Physics, QTF Centre of Excellence, Aalto University, FI-00076 AALTO, Finland
}

(Received 15 January 2021; accepted 20 May 2021; published 2 July 2021)

\begin{abstract}
A magnon Bose-Einstein condensate (BEC) in superfluid ${ }^{3} \mathrm{He}$ is a fine instrument for studying the surrounding macroscopic quantum system. At zero temperature, the BEC is subject to a few distinct forms of decay into other collective excitations, owing to momentum and energy conservation in a quantum vacuum. We study the vortexHiggs mechanism: The vortices relax the requirement for momentum conservation, allowing the optical magnons of the BEC to transform into light Higgs quasiparticles. This facilitates a direct measurement of the dimensions of the $B$-phase double-core vortex, providing experimental access to elusive phenomena, such as the Kelvin wave cascade and core-bound Majorana fermions. Our paper expands the spectrum of possible interactions between magnetic quasiparticles in ${ }^{3} \mathrm{He}-B$ and lays the groundwork for building magnon-based quantum devices.
\end{abstract}

DOI: 10.1103/PhysRevResearch.3.L032002

One illuminating perspective to the ground state of a fermionic condensate, such as zero-temperature superfluid ${ }^{3} \mathrm{He}$, is to treat it as a quantum vacuum where moving objects interact with the excitations of the vacuum [1-5]. Various collective excitations, for example, magnetic quasiparticles (magnons), and topological defects, such as quantized vortices can be manipulated in this extremely pure environment. A Bose-Einstein condensate of optical magnons (magnon BEC), trapped within the superfluid, can be instrumented to probe objects in the system without influencing them [6-9]. This capacity has inspired suggestions to use the BEC to detect surface- or vortex-core-bound Majorana fermions [10,11] or the Kelvin wave cascade $[12,13]$. Both have so far remained elusive despite decades of active research. Changes in the BEC ground-state frequency as well as the population decay rate of the BEC can be devised for such purposes, provided the basic interactions between the excitations of the quantum vacuum are first thoroughly mastered. On the other hand, macroscopic quantum systems, such as BEC-based time crystals [14-16] provide a promising building block for quantum technologies, which rely on controlled nondestructive manipulation of the system. Such control can be accessed in the

\footnotetext{
*s.autti@lancaster.ac.uk

Published by the American Physical Society under the terms of the Creative Commons Attribution 4.0 International license. Further distribution of this work must maintain attribution to the author(s) and the published article's title, journal citation, and DOI.
}

superfluid vacuum by coupling the BEC to and decoupling it from available excitations selectively.

In superfluid ${ }^{3} \mathrm{He}$, the spin and orbital angular momenta of Cooper pairs are equal to one. In the $B$ phase, the relative spin-orbit symmetry is broken in addition to the emergence of a coherent phase, as described by a $3 \times 3$ complex orderparameter matrix $[17,18]$. The macroscopic spin and orbital momentum directions are connected by the spin-orbit rotation angle $\theta$ around axis $\hat{\boldsymbol{n}}$. In equilibrium, $\theta$ is equal to the Leggett angle $\theta_{\mathrm{L}} \approx 104^{\circ}$. The fermionic thermal excitations of this system have energy gap $\Delta_{\mathrm{B}}$ which at low temperatures is on the order of $k_{\mathrm{B}} T_{\mathrm{c}}$, where $k_{\mathrm{B}}$ is the Boltzmann constant and $T_{\mathrm{c}}$ is the superfluid transition temperature. At temperatures much below $T_{\mathrm{c}}$, the number of thermal excitations is reduced exponentially, creating a vacuum void of fermionic quasiparticles.

Besides the fermionic quasiparticles, there are three collective spin-wave modes with a small (or zero) gap, corresponding to the combined oscillations of three spin components and three components of spin-orbit rotation [18]. Following Ref. [19], we call these modes optical magnons, acoustic magnons, and light Higgs quasiparticles. A detailed derivation of the modes can be found, e.g., in Ref. [20]. In the absence of a magnetic field, optical and acoustic magnons are gapless, corresponding to the oscillations of $\hat{\boldsymbol{n}}$. That is, their frequency vanishes in the long-wavelength limit. In a magnetic field $H$, optical magnons acquire a gap equal to the Larmor frequency $2 \pi f_{\mathrm{L}}=\omega_{\mathrm{L}}=|\gamma| H$, where $\gamma$ is the gyromagnetic ratio. The light Higgs mode corresponds to oscillation of $\theta$ around $\theta_{\mathrm{L}}$ and has a gap $\Omega_{\mathrm{B}} / 2 \pi \sim 100 \mathrm{kHz}\left(\Omega_{\mathrm{B}}\right.$ is the Leggett frequency). The dispersion relations of these three modes are illustrated in Fig. 1(a). Direct interactions between these modes are studied in Ref. [19]. 
(a)

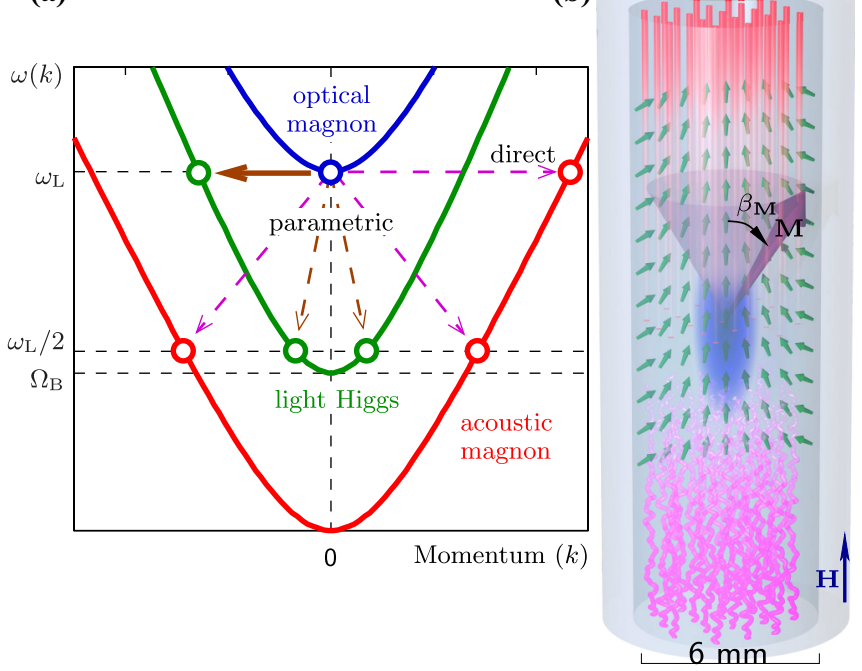

FIG. 1. (a) Spectra of spin waves in ${ }^{3} \mathrm{He}-\mathrm{B}$. The mass (gap) $\omega_{\mathrm{L}}$ of optical magnons (blue line) can be tuned using the magnetic field. Direct conversion of optical magnons into light Higgs quasiparticles (solid arrow), studied in this Letter, requires balancing the momentum mismatch. The parametric conversion of optical magnons into gapless acoustic magnons (red line), and light Higgs quasiparticles (green line) can be observed when the density and mass of the optical magnons are large. Processes indicated by dashed arrows were reported in Ref. [19]. (b) Superfluid ${ }^{3} \mathrm{He}$ in a cylindrical container. A BEC of optical magnons (blue blob) is trapped in the middle by the spatial distribution of the orbital order parameter $(\hat{\boldsymbol{n}}$ vector, small green arrows) and by an axial minimum in the external magnetic field $\boldsymbol{H}$. The coherently precessing magnetization $\boldsymbol{M}$ (large magenta arrow) in the BEC is parametrized with the tipping angle $\beta_{\boldsymbol{M}}$. In constant rotation $\Omega$ around the vertical axis, an array of vortices is created, penetrating the BEC (red vertical rods at the top). For illustrational reasons the vortex rods have been made transparent in the vicinity of the BEC and drawn only in the upper half of the container, and the order parameter distribution is only shown in the middle section. The vortex configuration obtained in modulated rotation is sketched with the magenta rods at the bottom of the container, based on Ref. [21].

The superfluid vacuum can also host topological defects [1], in particular, quantized vortices. An ordered array of vortices can be created by rotating the sample at a constant angular velocity $\Omega$. The density of the vortex array is proportional to $\Omega$. The $B$-phase vortices have a broken-symmetry core $[22,23]$ where the low-temperature vortex studied in this Letter has a double-core structure consisting of two tightly bound half cores [24-30]. A theoretical description of the interactions of vortices and collective excitations in superfluid ${ }^{3} \mathrm{He}$ can be confidently constructed by expanding on the BCS theory with Fermi-liquid effects included [31].

In this Letter we study the interaction of a condensate of optical magnons with quantized vortices and light Higgs quasiparticles, which we call the vortex-Higgs mechanism: If a vortex penetrates the magnon BEC, optical magnons are scattered by the order-parameter distortion that surrounds the vortex. This interaction lifts the requirement for momentum conservation for inbound and outbound quasiparticles. We show that in such collisions the optical magnons in the con- densate are converted directly into light Higgs quasiparticles. This is seen as zero-temperature relaxation of the BEC. We study this conversion in two qualitatively different vortex configurations, ordered and disordered, and find that the results are in good agreement with theory.

The magnon BEC in superfluid ${ }^{3} \mathrm{He}$ consists of coherent optical magnons [32]. Their magnetization $\boldsymbol{M}$ precesses around the external magnetic field $\boldsymbol{H}$ and is described by a macroscopic wave function $\Psi$. The total number of magnons $N \propto \int|\Psi|^{2} d V \propto \int \beta_{M}^{2} d V$, where $\beta_{\boldsymbol{M}}$ is the deflection angle of $\boldsymbol{M}$ from the equilibrium direction along $\boldsymbol{H}$, and $V$ is the volume. Here we assumed that $\beta_{M}$ is small, which is satisfied in all the experiments presented in this Letter. The coherently precessing magnetization is generated and detected using nuclear magnetic resonance techniques: Transverse coils placed in the vicinity of the sample container cylinder allow driving the condensate using a radio-frequency field and detecting the condensate by measuring the induced voltage (Fig. S1a in the Supplemental Material [33]).

The magnon BEC is trapped in the middle of the superfluid sample [Fig. 1(b)] by the radial distribution of the orbital order parameter ("texture"), and an axial minimum of the external magnetic field. The resulting trap is nearly harmonic [34], characterized by the radial and axial trapping frequencies $f_{\mathrm{r}}$ and $f_{\mathrm{z}}$ determined from measurements of the full spectrum of states in the trap [20]. We concentrate on the ground-state magnon BEC, whose precession frequency is $f=f_{\mathrm{L}}+f_{\mathrm{r}}+$ $f_{\mathrm{z}} / 2$. Temperature is measured using a mechanical oscillator, a quartz tuning fork. Its resonance width follows $\Delta v \propto$ $\exp \left(-\Delta_{\mathrm{B}} / k_{\mathrm{B}} T\right)$ at temperatures $T \ll T_{\mathrm{c}} \sim 1 \mathrm{mK}$, probing the density of thermal quasiparticles [35]. Further details of the experimental setup can be found in the Supplemental Material [33].

At a finite temperature, the relaxation of a magnon BEC is primarily caused by spin transfer via thermal fermionic quasiparticles [34,36] via the Leggett-Rice spin-rotation effect [37]. This process is usually called nonhydrodynamical spin diffusion. It results in exponential decay of the condensate $\beta_{M} \propto \exp \left(-t / \tau_{\mathrm{SD}}\right)$. Here $1 / \tau_{\mathrm{SD}}$ is the spin-diffusion relaxation rate. The rate is proportional to the thermal quasiparticle density and, thus, to the thermometer fork resonance width and the BEC relaxation rate $1 / \tau_{\mathrm{SD}} \propto \Delta v$ [red points in Fig. 2(a)] [34]. In practice there are also unavoidable losses in the measurement circuitry, but this effect can be confidently subtracted [34].

In the zero-temperature limit intrinsic decay channels are absent, and the condensate lifetime approaches infinity $[7,14,16,38]$. Any (extrapolated) zero-temperature dissipation in the bulk liquid [38] is, therefore, an indication of interaction with other collective modes either via parametric excitation or via direct conversion. The former is allowed assuming the density of optical magnons is high enough, and their mass is large enough [19]. Direct conversion is ruled out due to momentum conservation unless mediated by boundaries or interaction with topological defects of the superfluid vacuum, such as quantum vortices.

The interaction with a nonsingular topological defect arises due to the distortion of the order parameter distribution in the vicinity of the defect: The order parameter is not suppressed to zero in the core but changes to another superfluid phase. 

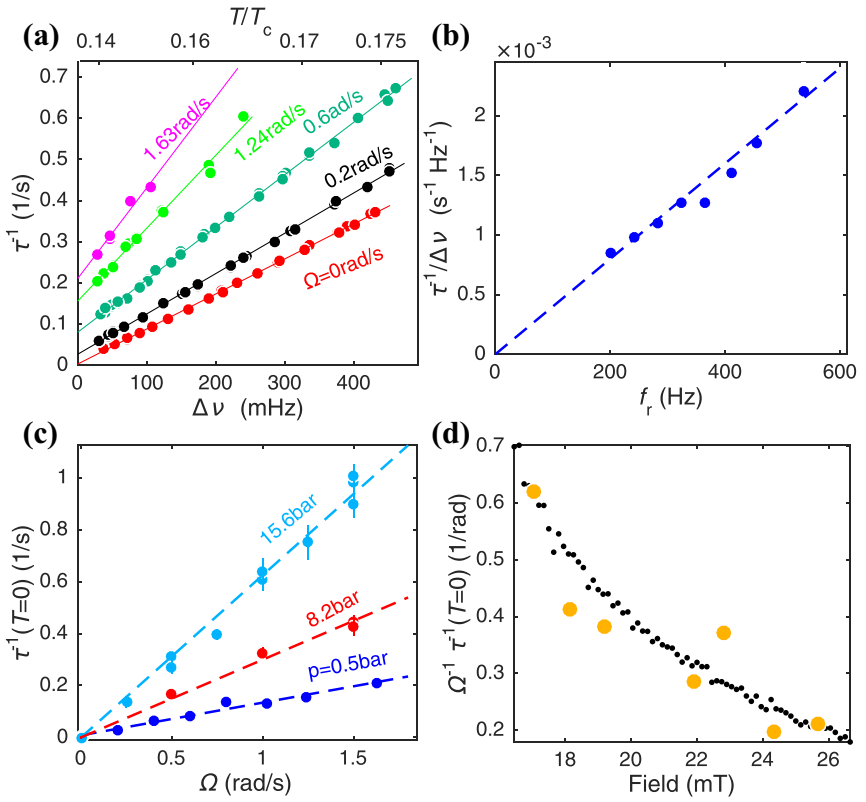

FIG. 2. Vortex-Higgs mechanism in steady rotation: (a) Measured relaxation rate at $\Omega=0$ (red points) is linear in the thermometer fork width $\Delta v$ owing to spin diffusion (red line). Intrinsic fork width has been subtracted from $\Delta v$ shown here. Data measured at $\Omega>0$ show an increased slope, reflecting the changing trap shape. (b) The temperature-dependence slopes $\frac{d \tau^{-1}(\Omega)}{d \Delta v}$ in panel (a) (points) are proportional to the radial trapping frequency $f_{\mathrm{r}}$ (dashed line is a linear fit through zero) as expected for spin diffusion, implying that other relaxation contributions are temperature independent. (c) The temperature-independent relaxation extracted as illustrated in panel (a) by extrapolating to $\Delta v=0$ (colored dots) is proportional to $\Omega$, that is, to the vortex density (dashed lines). (d) The magnetic-field dependence of the vortex relaxation in the ordered state, extracted similarly as shown in panel (a) (large orange circles, $\Omega=1 \mathrm{rad} \mathrm{s}^{-1}$ ), is in good agreement with that obtained by modulated rotation (small black points). Dissipation due to losses in the measurement circuity and the intrinsic fork width have been subtracted from all data as explained in Ref. [34] and Fig. 3. Pressure in panels (a) and (b) was 0.5 bar. The magnetic field for the 0.5 -bar and 15-bar data corresponds to $f_{\mathrm{L}}=826 \mathrm{kHz}$, and for the 4-bar and 8-bar data to $f_{\mathrm{L}}=833 \mathrm{kHz}$. Data in panel (d) were measured at 4-bar pressure. Error bars correspond to uncertainty in removing the resonant relaxation peaks.

In a simple model, the double-core vortex can be represented as a pair of half-quantum vortices [25,29] where the connection between the cores forms a domain wall. Domain walls, such as phase boundaries characteristically orient orbital degrees of freedom in ${ }^{3} \mathrm{He}$ in a distinct way as observed, e.g., across the boundary between the $A$ and $B$ phases of superfluid ${ }^{3} \mathrm{He}[36,39]$. Thus, the longer the connecting wall, the larger the distortion. Around the double-core vortex $\theta-\theta_{\mathrm{L}} \approx C / r$, where $C$ characterizes the strength of the distortion and $r$ is distance from the central axis of the vortex. This deviation becomes substantial $\left(\theta-\theta_{\mathrm{L}} \sim 1\right)$ at distances comparable to the half-core separation. Thus, $C$ measures this distance (Eq. (S4) in the Supplemental Material [33]). This deviation of $\theta$ leads to coupling between magnons in the BEC and light Higgs modes as is derived in Ref. [40] and applied for the present case in the Supplemental Material [33].
The entire refrigerator used in the experiments can be rotated around the axis of the sample container cylinder. Rotation creates an equilibrium array of vortices, which has a twofold effect on the BEC. First, the global orbital texture reacts to the vortex array [41], changing the shape of the trap ( $f_{\mathrm{r}}$ increases). This affects the spin-diffusion relaxation, which can be written as $1 / \tau_{\mathrm{SD}}=f_{\mathrm{r}} D \times$ const [36] $\left(f_{\mathrm{r}} \gg f_{\mathrm{z}}\right)$. Here $D$ is the applicable component of the transverse spin-diffusion tensor. We find that changes in the measured relaxation $1 / \tau$ are proportional to $\Delta v$ at any given $\Omega$ [Fig. 2(a)] and that the slope $\frac{d \tau^{-1}(\Omega)}{d \Delta v}$ is proportional to the measured radial trapping frequency $f_{\mathrm{r}}$ [Fig. 2(b)]. This observation implies that the temperature dependence of the relaxation rate $1 / \tau$, contained in $D$, is not affected by rotation, and any relaxation directly related to the vortices is temperature independent below $T=0.17 T_{\mathrm{c}}$. We emphasize that all the relaxation signals measured were exponential in time, implying that no nonexponential contribution was added by the vortex array. The second observation is that the zero-temperature $(\Delta v=0)$ extrapolation of the relaxation is proportional to $\Omega$ [Fig. 2(c)]. That is, the observed temperature-independent relaxation is as follows: (i) exponential and (ii) proportional to the density of vortices (see Fig. S1b in the Supplemental Material [33]). This is in good agreement with the theoretical expectation for vortex-Higgs mechanism of BEC relaxation, Eq. (S17) of the Supplemental Material [33].

We note that peaks in the measured relaxation, associated with the presence of vortices, were observed with roughly $1-\mathrm{kHz}$ spacing in $f_{\mathrm{L}}$ on top of the vortex-Higgs dissipation described above. We account this phenomenon for resonant production of standing spin-wave modes in the sample container, mediated by the vortex array. In Ref. [19] such peaks are accounted to vortex-mediated production of acoustic magnons [see Fig. 1(a)]. We believe both acoustic magnons and light Higgs quasiparticles are viable candidates to explain this observation, but a detailed study is left for a future publication. For simplicity, in what follows we call the peaks relaxation peaks. The peak frequencies were avoided in all measurements conducted at stable rotation.

As an alternative to constant rotation, the angular velocity can be modulated. We used linear modulation in the range from 1.4 to $1.8 \mathrm{rad} \mathrm{s}^{-1}$ with $|d \Omega / d t|=0.03 \mathrm{rad} \mathrm{s}^{-2}$. In the steady state the vortex number is expected to remain constant but the vortex array is distorted. We find experimentally that this removes the resonance peaks found at constant rotation. The measured zero-temperature relaxation with and without modulation, the latter avoiding the relaxation peaks are shown in Fig. 2(d). The two vortex configurations yield the same $\mathrm{BEC}$ relaxation rate. This observation allows us to probe the vortex-Higgs mechanism at arbitrary magnetic fields, avoiding the relaxation peaks altogether.

We can further characterize the vortex-Higgs mechanism by varying the coupling among magnons and vortices and, thus, parameter $C$. The core separation can be varied by changing pressure. The relaxation caused by the vortex-Higgs mechanism also depends on the magnitude on the magnetic field as derived in the Supplemental Material [33].

We compare the experiment with the theoretical model in Fig. 3. We find very good agreement in the magnetic field as well as the pressure dependence with $C=7.4 R_{0}$. Here $R_{0}$ 


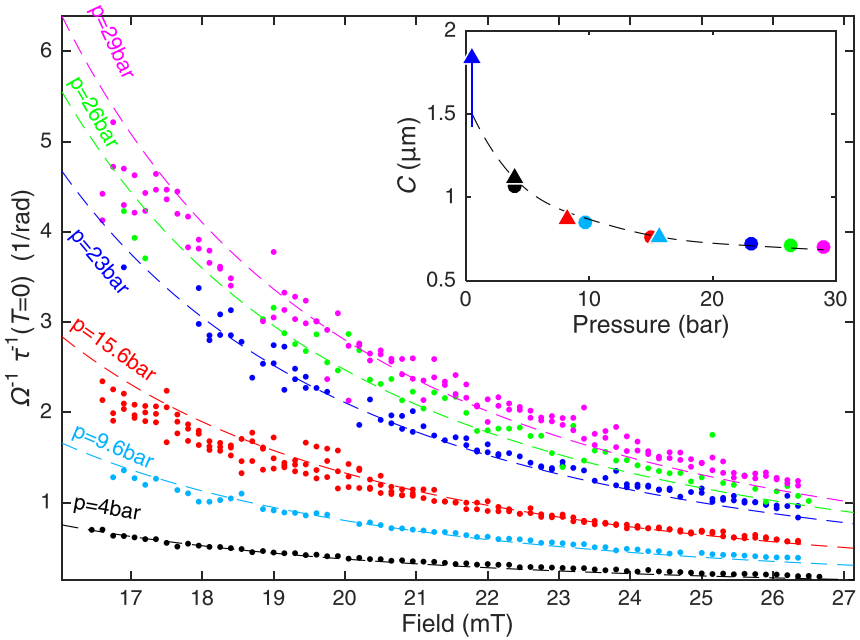

FIG. 3. Vortex-Higgs mechanism as a function of magnetic field and pressure: Measured BEC-relaxation field dependence at different pressures (colored dots) is in good agreement with the theoretical expectation (dashed lines) for vortex-mediated conversion of BEC magnons into light Higgs quasiparticles. Theory lines correspond to Eq. (S17) in the Supplemental Material [33], fitted to the data using parameter $C$. All measurements were carried out at $T=0.15 T_{\text {c }}$. Spin-diffusion dissipation and radiation losses have been subtracted based on measured trapping frequencies $f_{\mathrm{r}}$ and $f_{\mathrm{z}}$ [36]. This correction is about $5 \%$ for the 4-bar data and less than $1 \%$ for the 29-bar data. The inset shows $C$ vs pressure, in good agreement with theoretical expectation $C=\tilde{C} R_{0}$ with fitted $\tilde{C}=7.4$ (dashed line). The colored circles correspond to fits in the main panel, and the triangles correspond to the straight-vortex data in Figs. 2(c) and 2(d). Error bars correspond to uncertainty in removing the resonant relaxation peaks.

is a characteristic length scale on the order of the coherence length in the superfluid (see Supplemental Material [33] for details). This value is close to the theoretical value of $C / R_{0}=$ 5.9-6.6 [27,42], and, therefore, the agreement between experiment and theory is highly satisfactory without any fitting parameters. This means that the fitted $C$ agrees well with the theoretically predicted core separation. The millimeter-sized magnon BEC, therefore, correctly measured the effective vortex half-cores' separation which is on the order of $1 \mu \mathrm{m}$, confirming the assumption that the BEC interacts with each individual vortex independently and providing a strong argument in support of the vortex-Higgs interpretation.

The observations presented above imply that the vortexHiggs mechanism opens an otherwise-unavailable relaxation channel for the magnon BEC, corresponding to zerotemperature conversion of optical magnons of the BEC into light Higgs quasiparticles. This connection is mediated by the double-core vortex of low-temperature superfluid ${ }^{3} \mathrm{He}$, and it is robust against changes in vortex orientation and order. The role of the vortices, acting via the textural distortion that surrounds them, is to bridge the mismatch of momentum between the two species of quasiparticles. The measured dissipation is proportional to vortex density and reacts to changes in the core dimensions of the $B$-phase vortices and the order parameter distribution surrounding them as expected from the theory developed in the Supplemental Material [33]. These observa- tions add the vortex-Higgs mechanism to the set of confirmed interaction channels of magnetic quasiparticles in superfluid ${ }^{3} \mathrm{He}$.

It remains an interesting task for the future to confirm further predictions of the vortex-Higgs mechanism. The conversion of optical magnons into light Higgs quasiparticles is only possible via this mechanism assuming the optical magnons have a mass $\left(\propto \omega_{\mathrm{L}}\right)$ larger than that of light Higgs quasiparticles $\left(\propto \Omega_{\mathrm{B}}\right)$. The former is controlled by the magnetic field, and the latter by pressure. Probing the region where $\omega_{\mathrm{L}}<\Omega_{\mathrm{B}}$, and, thus, the vortex-Higgs mechanism is disabled, requires developing a spectrometer capable of measuring at a sufficiently low magnetic field $(<8 \mathrm{mT})$. Such a setup could be used to conclusively identify the source of the resonant relaxation peaks as well. On the other hand, our results provide basis for looking for relaxation contributions beyond the vortex-Higgs mechanism, for example, those originating from vortex-core-bound or surface-bound fermionic quasiparticles $[10,11,43-45]$. For such studies one should also operate below the cutoff frequency $\Omega_{\mathrm{B}}$, allowing the detection of smaller relaxation contributions.

The magnon BEC also makes a sophisticated new tool for probing other emergent phenomena, such as vortex dynamics. In particular, zero-temperature vortex turbulence is believed to be terminated by the Kelvin wave cascade, but it remains a long-standing challenge to confirm and explore this effect experimentally $[12,13,46,47]$. On the other hand, surfaces and vortex cores of superfluid ${ }^{3} \mathrm{He}$ host elusive Majorana bound states $[10,11]$, with a characteristic zero-temperature dissipation signature [48], which remains to be conclusively evidenced. Both these realms can now be explored using a magnon BEC as an instrument. For example, one way of detecting the Majorana quasiparticles relies on changing the relative magnetic-field orientation and measuring the related magnetic relaxation [48]. Our Letter provides a solid basis for measuring any delicate field dependence of the BEC relaxation rate. Finally, applying the BEC to build quantum devices [16] — eventually perhaps even at room temperature [15,4952] - is an exciting new avenue for research that our Letter and other recent advances have enabled. Quantum vortices could be used to manipulate the state and features of such a device via the vortex-Higgs mechanism with minimal disturbance and coupling to the external world.

The data that support the findings in this Letter are available from the corresponding author upon reasonable request.

This work has been supported by the European Union's Horizon 2020 research and innovation programme (Grant No. 694248). The experimental work was carried out in the Low Temperature Laboratory, which is a part of the OtaNano research infrastructure of Aalto University and of the EU H2020 European Microkelvin Platform (Grant No. 824109). S.A. and V.V.Z. were funded by UK EPSRC (Grant No. EP/P024203/1). S.A. acknowledges support from the Jenny and Antti Wihuri Foundation, P.J.H. that from the Väisälä Foundation of the Finnish Academy of Science and Letters, and S.M.L. that from both of the above. E.V.T. acknowledges support by the Academy of Finland Centre of Excellence program (Project No. 312057). 
[1] G. E. Volovik, The Universe in a Helium Droplet (Oxford University Press, Oxford, 2003).

[2] D. I. Bradley, S. N. Fisher, A. M. Guénault, R. P. Haley, C. R. Lawson, G. R. Pickett, R. Schanen, M. Skyba, V. Tsepelin, and D. E. Zmeev, Breaking the superfluid speed limit in a fermionic condensate, Nat. Phys. 12, 1017 (2016).

[3] J. A. Kuorelahti, S. M. Laine, and E. V. Thuneberg, Models for supercritical motion in a superfluid fermi liquid, Phys. Rev. B 98, 144512 (2018).

[4] S. Autti, J. T. Mäkinen, J. Rysti, G. E. Volovik, V. V. Zavjalov, and V. B. Eltsov, Exceeding the landau speed limit with topological bogoliubov fermi surfaces, Phys. Rev. Research 2, 033013 (2020).

[5] S. Autti, S. L. Ahlstrom, R. P. Haley, A. Jennings, G. R. Pickett, M. Poole, R. Schanen, A. A. Soldatov, V. Tsepelin, J. Vonka, T. Wilcox, A. J. Woods, and D. E. Zmeev, dissipation due to bound fermions in the zero-temperature limit, Nat. Commun. 11, 4742 (2020).

[6] Y. M. Bunkov, S. N. Fisher, A. M. Guénault, and G. R. Pickett, Persistent Spin Precession in ${ }^{3} \mathrm{He}-\mathrm{B}$ in the Regime of Vanishing Quasiparticle Density, Phys. Rev. Lett. 69, 3092 (1992).

[7] S. N. Fisher, A. M. Guénault, A. J. Hale, G. R. Pickett, P. A. Reeves, and G. Tvalashvili, Thirty-minute coherence in free induction decay signals in superfluid ${ }^{3} \mathrm{He}-\mathrm{B}$, J. Low Temp. Phys. 121, 303 (2000).

[8] S. Autti, Y. M. Bunkov, V. B. Eltsov, P. J. Heikkinen, J. J. Hosio, P. Hunger, M. Krusius, and G. E. Volovik, Self-Trapping of Magnon Bose-Einstein Condensates in the Ground State and on Excited Levels: From Harmonic to Box Confinement, Phys. Rev. Lett. 108, 145303 (2012).

[9] S. Autti, V. V. Dmitriev, J. T. Mäkinen, J. Rysti, A. A. Soldatov, G. E. Volovik, A. N. Yudin, and V. B. Eltsov, Bose-Einstein Condensation of Magnons and Spin Superfluidity in the Polar Phase of ${ }^{3} \mathrm{He}$, Phys. Rev. Lett. 121, 025303 (2018).

[10] S. Murakawa, Y. Wada, Y. Tamura, M. Wasai, M. Saitoh, Y. Aoki, R. Nomura, Y. Okuda, Y. Nagato, M. Yamamoto, S. Higashitani, and K. Nagai, Surface Majorana cone of the superfluid ${ }^{3}$ He B phase, J. Phys. Soc. Jpn. 80, 013602 (2011).

[11] B. Rosenstein, I. Shapiro, and B. Y. Shapiro, Effect of nanoholes on the vortex core fermion spectrum and heat transport in $\mathrm{p}$ wave superconductors, J. Phys.: Condens. Matter 25, 075701 (2013).

[12] V. B. Eltsov and V. S. L'vov, Amplitude of waves in the Kelvinwave cascade, JETP Lett. 111, 389 (2020).

[13] V. S. L'vov and S. Nazarenko, Spectrum of Kelvin-wave turbulence in superfluids, JETP Lett. 91, 428 (2010).

[14] S. Autti, V. B. Eltsov, and G. E. Volovik, Observation of a Time Quasicrystal and Its Transition to a Superfluid Time Crystal, Phys. Rev. Lett. 120, 215301 (2018).

[15] A. J. E. Kreil, H. Y. Musiienko-Shmarova, S. Eggert, A. A. Serga, B. Hillebrands, D. A. Bozhko, A. Pomyalov, and V. S. L'vov, Tunable space-time crystal in room-temperature magnetodielectrics, Phys. Rev. B 100, 020406(R) (2019).

[16] S. Autti, P. J. Heikkinen, J. T. Mäkinen, G. E. Volovik, V. V. Zavjalov, and V. B. Eltsov, AC Josephson effect between two superfluid time crystals, Nature Mater. 20, 171 (2021).

[17] A. J. Leggett, A theoretical description of the new phases of liquid He 3, Rev. Mod. Phys. 47, 331 (1975).

[18] D. Vollhardt and P. Wölfle, The Superfluid Phases of Helium 3 (Dover, New York, 2013).
[19] V. V. Zavjalov, S. Autti, V. B. Eltsov, P. J. Heikkinen, and G. E. Volovik, Light Higgs channel of the resonant decay of magnon condensate in superfluid ${ }^{3} \mathrm{He}$. Nat. Commun. 7, 10294 (2016).

[20] V. V. Zavjalov, S. Autti, V. B. Eltsov, and P. J. Heikkinen, Measurements of the anisotropic mass of magnons confined in a harmonic trap in superfluid ${ }^{3} \mathrm{He}-\mathrm{B}$, JETP Lett. 101, 802 (2015).

[21] J. T. Mäkinen and V. B. Eltsov, Mutual friction in superfluid ${ }^{3} \mathrm{He}-\mathrm{B}$ in the low-temperature regime, Phys. Rev. B 97, 014527 (2018).

[22] M. M. Salomaa and G. E. Volovik, Quantized vortices in superfluid ${ }^{3} \mathrm{He}$, Rev. Mod. Phys. 59, 533 (1987).

[23] O. V. Lounasmaa and E. V. Thuneberg, Vortices in rotating superfluid ${ }^{3} \mathrm{He}$, Proc. Natl. Acad. Sci. U.S.A. 96, 7760 (1999).

[24] E. V. Thuneberg, Ginzburg-Landau theory of vortices in superfluid ${ }^{3}$ He-B, Phys. Rev. B 36, 3583 (1987).

[25] G. E. Volovik, Half-quantum vortices in superfluid ${ }^{3} \mathrm{He}-\mathrm{B}$, Pis'ma Zh. Eksp. Teor. Fiz. 52, 972 (1990) [JETP Lett. 52, 358 (1990)].

[26] M. Fogelström and J. Kurkijärvi, Quasiclassical theory of vortices in ${ }^{3}$ He-B, J. Low Temp. Phys. 98, 195 (1995).

[27] M. A. Silaev, E. V. Thuneberg, and M. Fogelström, Lifshitz Transition in the Double-Core Vortex in ${ }^{3} \mathrm{He}-\mathrm{B}$, Phys. Rev. Lett. 115, 235301 (2015).

[28] K. Kasamatsu, R. Mizuno, T. Ohmi, and M. Nakahara, Effects of a magnetic field on vortex states in superfluid ${ }^{3} \mathrm{He}-\mathrm{B}$, Phys. Rev. B 99, 104513 (2019).

[29] N. Nagamura and R. Ikeda, Double-core vortex stabilized by disorder in superfluid ${ }^{3} \mathrm{He} \mathrm{B}$ phase in globally isotropic aerogel, arXiv: 1905.02569.

[30] R. C. Regan, J. J. Wiman, and J. A. Sauls, Vortex phase diagram of rotating superfluid ${ }^{3} \mathrm{He}-\mathrm{B}$, Phys. Rev. B 101, 024517 (2020).

[31] J. W. Serene and D. Rainer, The quasiclassical approach to superfluid ${ }^{3} \mathrm{He}$, Phys. Rep. 101, 221 (1983).

[32] Y. M. Bunkov and G. E. Volovik, Novel Superfluids (Oxford University Press, Oxford, 2013), Vol. 1, pp. 253-311.

[33] See Supplemental Material at http://link.aps.org/supplemental/ 10.1103/PhysRevResearch.3.L032002 for details on the experimental techniques and a derivation of the vortex-Higgs mechanism. The Supplemental Material includes Refs. [52-55].

[34] P. J. Heikkinen, S. Autti, V. B. Eltsov, J. J. Hosio, M. Krusius, and V. V. Zavjalov, Relaxation of Bose-Einstein condensates of magnons in magneto-textural traps in superfluid ${ }^{3} \mathrm{He}-\mathrm{B}$, J. Low Temp. Phys. 175, 3 (2014).

[35] R. Blaauwgeers, M. Blažková, M. Človečko, V. B. Eltsov, R. de Graaf, J. Hosio, M. Krusius, D. Schmoranzer, W. Schoepe, L. Skrbek, P. Skyba, R. E. Solntsev, and D. E. Zmeev, Quartz tuning fork: Thermometer, pressure- and viscometer for helium liquids, J. Low Temp. Phys. 146, 537 (2007).

[36] P. J. Heikkinen, S. Autti, V. B. Eltsov, R. P. Haley, and V. V. Zavjalov, Microkelvin thermometry with Bose-Einstein condensates of magnons and applications to studies of the $\mathrm{AB}$ interface in superfluid ${ }^{3} \mathrm{He}$, J. Low Temp. Phys. 175, 681 (2014).

[37] D. Einzel, The spin diffusion in normal and superfluid fermi liquids, J. Low Temp. Phys. 84, 321 (1991).

[38] S. N. Fisher, G. R. Pickett, P. Skyba, and N. Suramlishvili, Decay of persistent precessing domains in ${ }^{3} \mathrm{He}-\mathrm{B}$ at very low temperatures, Phys. Rev. B 86, 024506 (2012). 
[39] M. Arrayás, R. P. Haley, G. R. Pickett, and D. Zmeev, Orbitropic effect in superfluid ${ }^{3} \mathrm{He}$ B-phase boundaries, Sci. Rep. 8, 13965 (2018).

[40] S. M. Laine and E. V. Thuneberg, Spin-wave radiation from vortices in ${ }^{3} \mathrm{He}-\mathrm{B}$, Phys. Rev. B 98, 174516 (2018).

[41] V. B. Eltsov, R. De Graaf, M. Krusius, and D. E. Zmeev, Vortex core contribution to textural energy in ${ }^{3} \mathrm{He}-\mathrm{B}$ below $0.4 \mathrm{~T}_{\mathrm{c}}$, J. Low Temp. Phys. 162, 212 (2011).

[42] M. A. Silaev, E. V. Thuneberg, and M. Fogelström (unpublished).

[43] M. A. Silaev, Universal Mechanism of Dissipation in Fermi Superfluids at Ultralow Temperatures, Phys. Rev. Lett. 108, 045303 (2012).

[44] N. B. Kopnin and M. M. Salomaa, Mutual friction in superfluid ${ }^{3} \mathrm{He}$ : Effects of bound states in the vortex core, Phys. Rev. B 44, 9667 (1991).

[45] N. B. Kopnin and G. E. Volovik, Rotating vortex core: An instrument for detecting core excitations, Phys. Rev. B 57, 8526 (1998).

[46] E. Kozik and B. Svistunov, Kelvin-Wave Cascade and Decay of Superfluid Turbulence, Phys. Rev. Lett. 92, 035301 (2004).

[47] D. Kivotides, J. C. Vassilicos, D. C. Samuels, and C. F. Barenghi, Kelvin Waves Cascade in Superfluid Turbulence, Phys. Rev. Lett. 86, 3080 (2001).

[48] S. B. Chung and S.-C. Zhang, Detecting the Majorana Fermion Surface State of ${ }^{3} \mathrm{He}-\mathrm{B}$ Through Spin Relaxation, Phys. Rev. Lett. 103, 235301 (2009).
[49] A. J. E. Kreil, A. Pomyalov, V. S. L'vov, H. Y. MusiienkoShmarova, G. A. Melkov, A. A. Serga, and B. Hillebrands, Josephson oscillations in a room-temperature Bose-Einstein magnon condensate, arXiv:1911.07802.

[50] D. A. Bozhko, A. J. E. Kreil, H. Y. Musiienko-Shmarova, A. A. Serga, A. Pomyalov, V. S. L'vov, and B. Hillebrands, Bogoliubov waves and distant transport of magnon condensate at room temperature, Nat. Commun. 10, 2460 (2019).

[51] A. J. E. Kreil, D. A. Bozhko, H. Y. Musiienko-Shmarova, V. I. Vasyuchka, V. S. L'vov, A. Pomyalov, B. Hillebrands, and A. A. Serga, From Kinetic Instability to Bose-Einstein Condensation and Magnon Supercurrents, Phys. Rev. Lett. 121, 077203 (2018).

[52] D. A. Bozhko, A. A. Serga, P. Clausen, V. I. Vasyuchka, F. Heussner, G. A. Melkov, A. Pomyalov, V. S. L'Vov, and B. Hillebrands, Supercurrent in a room-temperature Bose-Einstein magnon condensate, Nat. Phys. 12, 1057 (2016).

[53] P. J. Heikkinen, Magnon Bose-Einstein condensate as a probe of topological superfluid, Ph.D. thesis, Aalto University School of Science, 2016, https://aaltodoc.aalto.fi/handle/123456789/ 20580.

[54] M. Silveri, T. Turunen, and E. Thuneberg, Hard domain walls in superfluid ${ }^{3} \mathrm{He}-\mathrm{B}$, Phys. Rev. B 90, 184513 (2014).

[55] E. V. Thuneberg, Hydrostatic theory of superfluid ${ }^{3} \mathrm{He}-\mathrm{B}$, J. Low Temp. Phys. 122, 657 (2001) 